\title{
Modulation of senoinflammation by calorie restriction based on biochemical and Omics big data analysis
}

\author{
EunJin Bang ${ }^{1}$, Bonggi Lee ${ }^{2}$, Sang-Gyun Noh ${ }^{1}$, Dae Hyun Kim ${ }^{1}$, Hee Jin Jung ${ }^{1}$, Sugyeong Ha ${ }^{1}$, Byung Pal Yu ${ }^{3} \mathcal{E}$ \\ Hae Young Chung, \\ ${ }^{1}$ Department of Pharmacy, College of Pharmacy, Pusan National University, Busan $46241,{ }^{2}$ Korean Medicine (KM)-Application Center, \\ Korea Institute of Oriental Medicine (KIOM), Daegu 41062, Korea, ${ }^{3}$ Department of Physiology, The University of Texas Health Science \\ Center at San Antonio, TX 78229, USA
}

\begin{abstract}
Aging is a complex and progressive process characterized by physiological and functional decline with time that increases susceptibility to diseases. Aged-related functional change is accompanied by a low-grade, unresolved chronic inflammation as a major underlying mechanism. In order to explain aging in the context of chronic inflammation, a new integrative concept on age-related chronic inflammation is necessary that encompasses much broader and wider characteristics of cells, tissues, organs, systems, and interactions between immune and non-immune cells, metabolic and non-metabolic organs. We have previously proposed a novel concept of senescent (seno)-inflammation and provided its frameworks. This review summarizes senoinflammation concept and additionally elaborates modulation of senoinflammation by calorie restriction (CR). Based on aging and CR studies and systems-biological analysis of Omics big data, we observed that senescence associated secretory phenotype (SASP) primarily composed of cytokines and chemokines was notably upregulated during aging whereas CR suppressed them. This result further strengthens the novel concept of senoinflammation in aging process. Collectively, such evidence of senoinflammation and modulatory role of CR provide insights into aging mechanism and potential interventions, thereby promoting healthy longevity. [BMB Reports 2019; 52(1): 56-63]
\end{abstract}

\section{INTRODUCTION}

Aging is a biological process characterized by time-dependent, progressive, and physiological functional decline that leads to increasing occurrence of age-related diseases. It is notable that

${ }^{*}$ Corresponding author. Tel: +82-51-510-2814; Fax: +82-51-5182821; E-mail: hyjung@pusan.ac.kr

https://doi.org/10.5483/BMBRep.2019.52.1.301

Received 23 October 2018

Keywords: Aging, Chronic inflammation, CR, Omics big data, Senoinflammation pro-inflammatory mediator genes are activated and commonly observed during the aging process (1). As generally accepted, chronic inflammation is the major underlying course of many age-related diseases. We have recently proposed new concept of senoinflammation to provide molecular evidence that link normal aging to pathological aging processes (2). Chronic inflammation in aging process occurs in a low-grade for a prolonged period of time with activation of macrophage and lymphocyte-accumulated leukocytes (3). During chronic inflammatory response, both destructive and repair programs driven by redox and cell death signaling pathways occur to remodel defective cells and tissues. This chronic inflammation is closely associated with alternation of redox state which has a causal relationship with inflammation. The delicate interplay between inflammation and redox state leads to aging and age-related inflammatory diseases.

In order to explain the diversity and complex aging phenomena, we designed, analyzed, and integrated Omics big data based on biological process and signaling pathways underlying the aging process. Using systems-biological tools, we systematically predicted and confirm that senoinflammation is involved in the aging process based on big data analysis of diverse molecular networks and signaling pathways.

In a previous report, we have proposed a novel concept of senoinflammation and its frameworks (2). Recent biochemical studies on calorie restriction (CR) which is widely accepted as a positive control of aging intervention and Omics big data analysis have shown low levels of inflammatory biomarkers, consistent with the basic tenet of the newly proposed senoinflammation concept. In this review, we attempted to introduce broadened overview of senoinflammation and its evidence at molecular level by examining various oxidatively altered senescence-associated secretory phenotypes (SASP) and its modulated signaling pathways based on CR studies which was further supported by Omics big data analysis.

\section{AGING PROCESS AND AGE-RELATED INFLAMMATION}

Aging is described by cognitive impairments and physiological

ISSN: 1976-670X (electronic edition)

Copyright (c) 2019 by the The Korean Society for Biochemistry and Molecular Biology

(ㄷ) This is an open-access article distributed under the terms of the Creative Commons Attribution Non-Commercial License (http://creativecommons.org/licenses/by-nc/4.0) which permits unrestricted non-commercial use, distribution, and reproduction in any medium, provided the original work is properly cited. 
functional decline that lead to morbidity and mortality. The aging is a complex phenomenon that is regulated by various cellular and biochemical processes. The oxidative stress hypothesis provides a molecular mechanistic explanation of aging, in which physiological functional degradation in aging is due to unceasing oxidative stress and compromised anti-oxidative defense system that eventually results in biological redox imbalance in the body (4). Therefore, major characteristics of age-related dysfunction are due to incapacity to properly regulate reactive species (RS) and maintain redox homeostasis, leading to chronic inflammation and age-related diseases.

Inflammation is described by a common biological immune response to pathogenic factors in immune system. It is triggered by stimulus of viral, fungal, and bacterial origins. Chronic inflammation is an unresolved, low-grade inflammatory response of the innate immune system. It may result in constant inflammatory response and abnormal innate immune response. In chronic inflammation, additional recruitment of immune cells such as macrophages and T cells is induced. This replaces and changes the initial neutrophil population occurred in acute inflammation. It is considered as a major underlying risk factor for aging and age-related diseases. Accumulating evidence supports that this systemic chronic inflammation plays an important role in regulating age-related disease such as dementia, obesity, type 2 diabetes, atherosclerosis, osteoporosis, sarcopenia, and others. Although the precise molecular inflammatory involvement of each disease may vary, basic mechanisms of induction and activation of cytokines and other pro-inflammatory mediators are similar.

In age-related chronic inflammation, a diverse array of cellular molecules and immune responses contribute to the aging process. The NLRP3 inflammasome, which has been linked to inflammatory diseases, is an intracellular multi-protein complex that recognizes various pathogenic, inflammationinducing stimuli such as pathogen-associated molecular patterns (PAMPs) and danger-associated molecular patters (DAMPs) responsible for the activation of pro-inflammatory responses. The NLRP3 inflammasome activation promotes secretion of IL-18 and inactive premature pro-IL-1 $\beta$ that matures into IL-1 $\beta$ which is a central regulator of the inflammatory response (5). It has been recently reported that suppression of NLRP3 inflammasome prolongs lifespan in vivo model by attenuating age-related degenerative changes including cognitive decline, suggesting that suppression of aberrant NLRP3 activity during aging may attenuate age-related diseases by alleviating chronic inflammation (6).

\section{AGE-RELATED INFLAMMATION AND SASP}

Cellular senescence is a process characterized by a permanent cell cycle arrest in response to senescence-inducing stressors. Cellular senescence importantly contributes to aging and age-related diseases. Senescent cells could exert serious and deleterious effects that hinder various normal cellular functions and lead to a direction of promoting pathological conditions such as chronic inflammation, aging of immune system, and age-related oncogenic response (7). These deleterious effects of senescent cells are largely due to their release of pro-inflammatory mediators in response to intracellular and extracellular stimuli. Baker et al. (7) have reported that the removal of senescent cells by injecting senolytic agents biweekly can extend the median lifespan of mice by approximately $30 \%$. Pro-inflammatory mediators released by senescence cells are collectively called senescenceassociated (SA) secretome commonly referred to as SASP (8). At molecular level, NF-KB signaling is the major signaling pathway that plays a role in stimulating the release and increase of SASP molecules composed of several families of 40-80 factors, including cytokines, chemokines, growth factors, and proteases $(8,9)$. The most potent SA secretomic pro-inflammatory cytokines are IL-1 $\beta$, IL- 6 , and IL-8 induced by DNA damage, replicative exhaustion, and oncogenic stimuli in keratinocytes, melanocytes, monocytes, fibroblasts, and epithelial cells (10-12). Other pro-inflammatory components of SASP include matrix metalloprotease (MMP) family members that are consistently increased in most senescent cells. MMPs are known to function in regulation of inflammation-related activities, including modulation of cytokines and chemokines (13). Recently, several studies have reported SASP-regulating molecules such as PTBP1 and HSP90 $(14,15)$.

Based on previous studies, SASP signaling in senescent cells is associated with aging and age-related inflammation which induces a large secretion of pro-inflammatory proteins that contribute to chronic inflammation. In addition, it has been shown that several pro-inflammatory SASP mediators are increased in aged human normal tissues of cancer patients in accordance with our RNA-seq data analysis (TCGA) (unpublished data).

\section{ANTI-SENOINFLAMMATION OF CALORIE RESTRICTION (CR)}

$\mathrm{CR}$ that includes reduced calories but proper nutrition intake is the sole intervention known to date that can consistently delay biological aging process and extend both average and maximal lifespan (16). Animal studies have documented that CR not only can increase lifespan, but also benefit other processes to promote overall health (17). CR has been shown to decrease spontaneous chemical- and radiation-mediated tumorigenesis in various mouse models of cancer (18). The incidence of chronic nephropathy and cardiomyopathy is also delayed or suppressed by CR (19). Furthermore, CR can inhibit $\beta$-amyloid deposition and neurodegeneration in central nervous system and promote neurogenesis in animal models of Alzheimer's, Parkinson's, Huntington's diseases, and stroke $(20,21)$. 
The beneficial effects of CR have also been documented in human studies. CR can reduce body mass index and atherosclerosis risk factors including total cholesterol and low-density lipoprotein-c and increase high density lipoproteins (16). Risk of type 2 diabetes is also reduced by CR. Furthermore, psychological and behavioral responses are markedly improved by 6 -month CR which includes reduction in binge eating, depression, and disinhibition and increase of physical functioning (16). Collectively, CR can increase diverse biomarkers of longevity, demonstrating its anti-aging properties in human studies.

Although mechanisms underlying CR-mediated delay of aging process should be diverse, it has been suggested that CR can enhance longevity and reduce pathology partially through its action on immune system as immune function decreases with aging while CR seems to be able to reverse this effect (22). It is well-known that the immune system of mammalian organisms has various alterations with aging that usually result in a decreased immune competence termed "immunosenescence" (22). It has been indicated that CR can regulate immune function and restore or delay immunosenescence (22). Although the mechanism underlying CR-mediated delay of immunosenescence remains to be elucidated, we speculated that CR might exert anti-immunosenescence effects through downregulation of senoinflammatory process.

\section{Inhibitory effects of CR on sasp expression in tissues}

Emerging evidence has shown that CR can markedly alleviate systemic inflammation due to its anti-inflammatory properties in various tissues. In white adipose tissue, CR markedly decreased mRNA expression levels of inflammatory cytokines and chemokines including IL-6, IL-1Ra, IL-2, MCP-1, and CXCL16 $(23,24)$. It increased mRNA levels of anti-oxidant catalase, superoxide dismutase 1 , and glutathione peroxidase 1 in obese mice (24). In liver, even mild CR decreased inflammatory gene expression such as MCP-1 and lipogenic gene expressions including SREBPs and PPARY (25). Anti-inflammatory effects of CR have also been shown in non-metabolic tissues. CR suppressed reactive species, lipid peroxides, and COX-2 activity in aged rat kidney. CR also inhibited upstream signaling cascades and DNA binding activity of NF-kB and AP-1 and thioredoxin/Ref-1 pathway. CR blocked expression of NF-kB-and AP-1-responsive gene COX-2, iNOS, VCAM-1, and ICAM-1 (26). Transient 3-week $\mathrm{CR}$ between birth and weaning period extended lifespan and led to permanent alterations in energy homeostasis, leptin, and insulin sensitivity (27). These beneficial effects by transient CR are associated with decrease in hypothalamic inflammation evidenced by decrease in markers of hypothalamic reactive gliosis related to aging such as GFAP-positive astrocytes and Iba1-positive microglia and reduction in age-associated overproduction of TNF $\alpha$ in microglia (27). Therefore, the anti-inflammatory effect of $C R$ is not limited to a specific tissue. Instead, CR can broadly reduce inflammation from central nervous system to peripheral tissues.

\section{Effects of CR on insulin sensitivity and ER stress}

It has been shown that CR can improve insulin sensitivity. When short-term CR (2-4 weeks) was applied to mice with metabolic syndrome induced by high calorie diet, mice exhibited improved glucose tolerance and hepatic insulin sensitivity (28). Long-term CR (3 months) ameliorated agingmediated systemic insulin resistance partially by improving insulin action in adipose tissues (29). There must be multiple underlying mechanisms of CR-mediated amelioration of insulin resistance in animal with metabolic syndrome attributed to aging or diet. Modulation of ER stress by CR partially accounts for this effect. A previous study showed that CR markedly decreased mRNA or protein expression levels of ER stress markers including activating transcription factor 4 , eukaryotic translation initiation factor $2 \alpha$, and phosphorylated PKR-like ER kinase in liver and epididymal fat of mice with metabolic syndrome (29). Furthermore, CR suppressed phosphorylation of insulin receptor substrate (IRS)-1 (Ser) and c-Jun $\mathrm{NH}(2)$-terminal kinase (JNK) known to be closely associated with ER stress $(30,31)$. Based on collected data, CR-mediated improvement of insulin resistance may be partially attributable to suppression of ER stress in metabolic tissues such as liver and adipose tissues.

\section{CR intervention on inflammasome formation}

Little has been known about the effect of CR on inflammasome formation. However, some studies have shown that systemic nutrient level might regulate inflammasome-mediated inflammatory response (32). When ischemic stroke was induced in C57BL/6 mice by middle cerebral artery occlusion, intermittent fasting reduced expression levels of NLRP1 and NLRP3 inflammasome proteins and IL- $1 \beta$ and IL-18 in ischemic brain tissue (33). In a human study, fasted individuals exhibited less NLRP3 inflammasome activation compared to individuals in refed condition, indicating that factors that could reduce nutrient level such as CR and fasting could suppress inflammasome formation (34). Although mechanisms underlying calorie deprivation-mediated suppression of inflammasome are not fully understood, an interesting study has revealed that the ketone body may be the intermediate product to inhibit inflammasome by $\mathrm{CR}$ or fasting. $\beta$-hydroxybutyrate $(\mathrm{BHB})$ is a ketone body produced by $\mathrm{CR}$ or fasting (32). BHB suppressed NLRP3 inflammasome by inhibiting $\mathrm{K}+$ efflux and decreasing the apoptosis-associated spec-like protein with a caspaserecruitment domain (ASC) oligomerization and speck formation (32). BHB decreased NLRP3 inflammasome-induced IL-1 $\beta$ and IL-18 production in human monocytes (32). These data suggest that anti-inflammatory effects of CR are likely to be associated with BHB-mediated suppression of NLRP3 inflammasome. 
Molecular modulation of anti-inflammatory action of CR

Although numbers of mechanisms underlying anti-aging effects of CR have been suggested to date, it is widely-accepted that anti-oxidative and anti-inflammatory properties of CR greatly contribute to its anti-aging effects (35). The most well-known anti-oxidative actions of $\mathrm{CR}$ are to inhibit cellular reactive species and control redox balance (35). Although intracellular sources of reactive species are diverse, the source of inflammation-associated reactive species is cyclooxygenase $(\mathrm{COX})$ that is essential for prostacyclin, prostaglandins, and thromboxane formation. CR can efficiently inhibit COXmediated reactive specie generation. This inhibition can be achieved at least partially by suppressing NF- $\mathrm{KB}$ activity that is responsible for expression of pro-inflammatory genes including inflammatory cytokines, inducible NO synthase, COX2, and others (36).

Another signaling pathway regulated by $\mathrm{CR}$ is MAPK signaling that is responsible for intracellular inflammatory reactions in response to diverse stimuli including oxidative stress. An animal study has shown that ERK, JNK, and p38 MAPK activities are highly increased in kidney of aged rats in parallel with increased ROS whereas CR can reverse these age-related activation of MAPKs, suggesting that regulation of MAPK signaling is necessary for mediation of beneficial anti-aging effect of CR (36).

In addition, it has been reported that CR could regulate PPAR activity (37). A previous study showed that gene expression levels and DNA binding activities of PPAR $\alpha$ and PPAR $\gamma$ were decreased in aged rat kidney whereas CR reversed such decreases (37). Upregulation of PPARs by $C R$ seems to play an important role in suppressing NF-KB

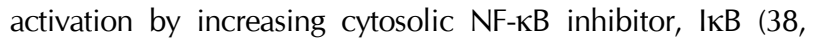
39). Consistently, PPAR $\alpha$ agonist treatment decreased pro-inflammatory cytokine production (39). Although more studies are necessary, it is likely that PPAR agonism can ameliorate aging-related inflammation through suppressing NF-кB-mediated inflammatory signaling (40).

It has been generally accepted that SIRT1 can extend lifespan and delay aging (41). SIRT1 is another key protein controlled by CR. It has been shown that CR can upregulate SIRT expression in liver, adipose tissue, brain, and kidney (42) and exert its anti-aging activity via interactions with FoxO1, FoxO3, FoxO4, PGC1 $\alpha$, p53, liver $\mathrm{X}$ receptor, and NF-kB. SIRT1-mediated deacetylation promotes nuclear trapping of FoxO1 and upregulates downstream gene expressions (43). Furthermore, SIRT1 deacetylates and inhibits NF-אB activation, consequently suppressing inflammatory cytokine production.

Emerging evidence supports that CR-mediated anti-aging and lifespan extension effects are mediated by suppressing senoinflammation which includes, but not limited to, production of SASP, insulin resistance, ER stress, and NLRP3 inflammasome formation. Molecular mechanisms underlying CR-mediated inhibition of senoinflammation (that is, anti-senoinflammation) involve inhibition of NF-KB signaling and activation of anti-inflammatory PPAR signaling.

\section{ANALYSIS OF AGING-RELATED OMICS BIG DATA}

\section{Evidence of senoinflammation concept based on Omics technologies and needs for further exploration}

Advances in Omics technologies have provided a technical breakthrough that enables one to investigate DNA, RNA, proteins, and other cellular molecules and their comprehensive interactions in biological context. One advantage of Omics approach is the use of high-throughput assays that can yield large amount of data on functional and structural alterations in complex systems. Nowadays, it is possible to analyze and integrate overall biological process that occurs in aging system at molecular level using state-of-the-art techniques such as next generation sequencing (NGS), proteomics, lipidomics, metabolics, and epigenomics. Furthermore, Omics big data analysis for signaling pathways and their combinations using bioinformatics and systems biology could be used as a powerful and informative tool.

We previously collected all microarray data for both aging and CR and further re-analyzed them using systems biology techniques based on computer science and algorithm. We collected and analyzed 478 aging- and 586 CR-related mouse genes. For given genes, biological pathways highly related to aging and CR were examined using gene ontology. Results demonstrated that gene expression patterns were highly related to immune response, lipid metabolism, and cell adhesion functions. In addition, investigation of transcriptional modularity analysis indicated that gene expressions of immune response and cell adhesion were upregulated while lipid metabolism-related genes were downregulated in the aging process. These changes were reversed by CR (44).

Five years later, we systems-biologically updated our agingrelated microarray data and found that inflammation-related genes were notably upregulated in the aging process while metabolism-related genes were downregulated by CR. In 2012, we re-analyzed microarray data of variable organs including metabolic organs using system biology method (45). As a result, we obtained 1,075 genes and CR transcriptome. Of them, 740 genes were expressed differentially in aging and 565 genes were differentially expressed in CR. Immune response-related genes were upregulated in aging and such upregulation was reversed by CR. Metabolism-related genes were downregulated in aging and such downregulation was also reversed by CR. Similar to previous studies, inflammationrelated genes were upregulated whereas metabolism-related genes such as PPAR and insulin signaling molecules were downregulated. Such up- and downregulations are modulated by CR. Using differentially expressed genes, we conducted a PPI network analysis. As a result, lymphocyte-specific protein tyrosinase kinase (LCK) was found as a hub protein showing high values of degree and betweenness centrality among other differentially expressed genes common to both aging and CR. 
Based on this study, we could infer that inflammation and metabolism are intimately related to each other from cellular level to system level in the aging process (45).

To better understand the regulatory role of inflammation in aging at molecular level, we sequenced the transcriptome of young and aged rat kidneys using RNA-seq to detect differentially regulated genes (46). Interestingly, cytokines, chemokines, and TNFs were significantly upregulated and TLRs-AKT/IKK-NFKB signaling pathway was activated in the aging process. Furthermore, we observed that genes related to amino acid, fatty acid metabolism, and PPAR $\alpha / \beta$ pathway were downregulated. RNA seq data of CR were recently analyzed. Results showed that JAK/STAT signaling pathway was decreased (unpublished data).

Our collaborators (Yoon, G group) have recently reviewed gene expressions in cell aging process using step-by-step microarray analysis. They reported significant upregulation of SASP-related genes such as cytokines, chemokines, growth factors, and MMPs-related molecules. It has been verified that inflammation-related SASP genes are upregulated in animal model at cellular level (47). Moreover, in order to examine effects of CR on age-related alterations in DNA methylation, we performed NGS analysis and examined the methylation of CpG islands in promoter regions of all genes using methylated DNA immunoprecipitation and next-generation sequencing (MeDIP-seq). It was found that inflammation-related genes were epigenomically regulated in the aging process such that $\mathrm{CpG}$ islands of inflammation and oncogene-related genes were hypomethylated. These changes are modulated by CR (48).

In addition, we further analyzed RNA seq, miRNA, mythylome in human normal tissue of cancer patients using big database (TCGA). We re-analyzed our data by age groups. We also examined gene expressions and epigenomic changes in the aging process. Interestingly, inflammation related cytokine/chemokine in JAK-STAT signaling pathway was upregulated in many organs and metabolic-related PPAR $\alpha / \beta$ signaling was downregulated. Similar biochemical results were obtained from cultured cell and animal experiments (unpublished data).

Based on Omics big data, JAK-STAT signaling was increased in both aged cells and tissues, thus increasing its downstream cytokines and chemokines molecules that were primary SASP. At the same time, factors such as DAMPs and PAMPs significantly activated IKK/Akt-NF-KB signaling pathway in aged tissues. By integrating and analyzing microarray data of various organs, we discovered that PPAR and insulin signaling-related genes were notably downregulated among other metabolism-related genes. We grouped altered signaling pathway in the aging process by metabolic organ and non-metabolic organ and further examined them using biochemical and molecular biological methods. As a result, ER stress and insulin resistance were increased, leading to hyperinsulinemia in metabolic organs. In non-metabolic organ, hyperinsulinemia induced activation of Akt-NF-KB signaling. This further caused inflammasome and inflammation $(49,50)$

\section{Construction of integrated signaling pathway related to inflammation and metabolism-related genes}

The integrative signaling pathway related to aging was performed using RNA-Seq analysis of rat kidney, microarray data analysis of metabolic organs such as mice and rat adipose tissue and muscle, RNA-seq analysis (TCGA) of human kidney, and proteomic data analysis of rat kidney. To establish a macromolecular biomolecular network associated with agerelated inflammation and metabolism, we have chosen

Table 1. Common pathways upregulated in aging based on Omics big data

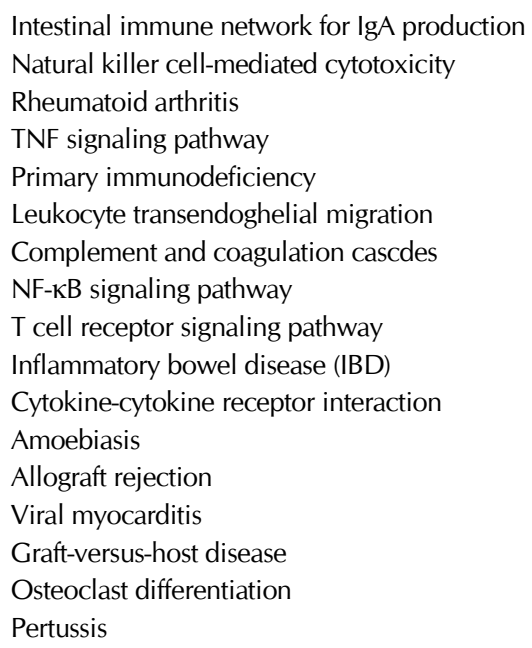

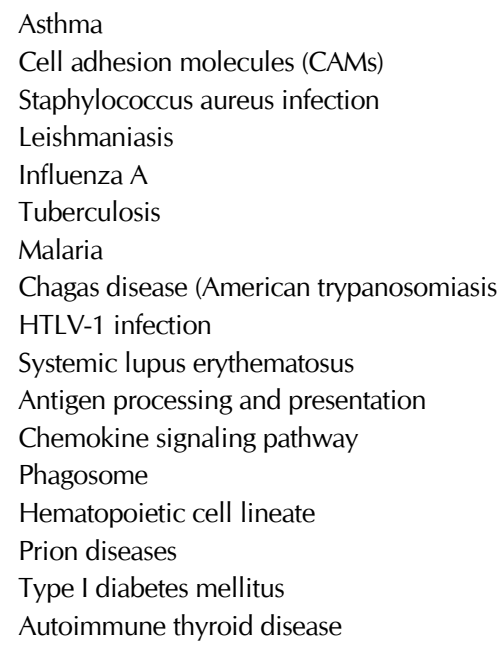


Table 2. List of genes upregulated in aging based on Omics big data

\begin{tabular}{cc}
\hline \multicolumn{1}{c}{ Pathway } & Genes \\
\hline Cytokine-cytokine receptor interaction & IL1R2, IL9R, CCL2, OSMR, TNFRSF12A, CCR1, TGFB3, TNFRSF8, CXCR2, CXCR3, CCL7, IL11, \\
& TGFB2, TNFRS1B, CCL20, CXCR4, CCL21, IL10RA, XCR1, CSF1R, IL18R1, IL6, IL23R, IL2RA, \\
& IL18RAP, TNFRS13B, TNFRS13C, EDA2R, CCL19, IL6R, TNFSF, CCL12, CCR7, TNFSF11, CCR6, \\
& CNTF, CCR5, CD40LG, CCR3 \\
Chemokine signaling pathway & CCL2, NCF1, CCR1, HCK, CXCL2, CCL19, CXCR2, CXCR3, CCL7, CCL6, CCL12, CCL22, CCR7, \\
NF-KB signaling pathway & CCR6, CCR5, CCL20, RAC2, CXCR4, CCL21, CCR3, PIK3R5, GNG2, XCR1, SHC2 \\
& TNFSF11, CD40LG, CCL21, LCK, ZAP70, TNFRSF13C, CCL19, LBP, BIRC3, BIK \\
\hline
\end{tabular}

Table 3. Common pathways downregulated in aging based on Omics big data

\begin{tabular}{lc}
\hline Pathway & Genes \\
\hline Biosynthesis antibiotics & AADAT, ACAA1B, ACAT1, ACAT2, ACLY, ACSS2, ACY1, ALDH1B1, ALDH7A1, ALDOA, CBS, CYP51, DLAT, \\
& EHH, ADH, ENO1, FBP1, FDPS, HSD17B7, IDI1, LSS, MDH2, NSDHL, ODC1, PCCA, PDHA1, PDHB, PFKL, \\
& PGM1, PHGDH, RGN, RPE, SQLE, TKT, TM7SF2 \\
Carbon metabolism & ACAT1, ACAT2, ACSS2, ALDOA, DLAT, EHHADH, ENO1, FBP1, MDH2, ME1, MTHFR, PC, PCCA, PDHA1, \\
Metabolic pathways & PDHB, PFKL, PHGDH, RGN, RPE, TKT \\
& AADAT, ACAA1B, ACACA, ACATI, ACAT2, ACLY, ACSS2, ACY1, AFMID, AGPAT2, AGPAT3, AKR1B1, \\
& AKR1B8, ALDH1B1, ALDH7A1, ALDOA, AMACR, CBS, CES1E, CNDP1, COASY, COMT, CRYL1, CYP4A3, \\
& CYP51, DEGS2, DGAT2, DHCR24, DHCR7, DLAT, EHHADH, ENO1, FASN, FBP1, FDPS, GATM, GBE1, \\
& GCLM, GCNT1, GPAM, GPHPR, HAAO, HSD11B1, HSD17B7, ID11, ITPK1, LAP3, LPIN1, LSS, LTC4S, \\
& MAN2A1, MAOB, MDH2, ME1, MECR, MTHFR, NATSL, NNT, NSDHL, ODC1, PC, PCCA, PCYT2, PDHA1, \\
& PDHB, PDHX, PFKL, PGM1, PHGDH, PHOSPHO1, PNP, PNPLA3, PTGIS, PYGL, RGN, RPE, SQLE, TKT, \\
Tyruvate metabolism & TM7SF2, UPP2, UROS \\
Tryptophan metabolism & ACACA, ACAT1, ACAT2, ACSS2, ALDH1B1, DLAT, GRHPR, MDH2, ME1, PC, PDHA1, PDHB \\
& AADAT, ACAT1, ACAT2, AFMID, ALDH1B1, EHHADH, MAOB \\
\hline
\end{tabular}

commonly detected pathway based on pathway analysis results from each Omics big data to identify biomarkers that are critical and central to aging research (Tables 1-3). We chose a commonly detected pro-inflammatory pathway based on the NGS platform and the microarray platform. The most commonly upregulated pathways were pro-inflammatory pathways including NF- $\mathrm{KB}$ signaling, cytokine-cytokine receptor interaction, chemokine signaling pathways, and others. The most commonly downregulated pathways were metabolic pathways including carbon metabolic pathway.

Taken together, these findings suggest that cytokine-cytokine receptor interaction and chemokine signaling pathway play an important role when inflammation increases in aging. Metabolic pathways and carbon metabolism also play an important role in decreasing metabolism in aging. Screening biomarker candidates involved in aging is expected to make significant progress in the study of aging in the future.

\section{CONCLUSION}

These dangerous effects of senescent cells are largely related to their release of pro-inflammatory mediators called SASP in response to extracellular and intracellular stimuli, leading to chronic inflammatory status called senoinflammation. Based on $\mathrm{CR}$ and Omics big data analysis, cytokine and chemokine pathway was notably upregulated in the aging process. CR generally accepted as a positive control of anti-aging intervention modulated and inhibited SASP secretion. Such evidence suggests that senoinflammation is an underlying molecular mechanism for aging process. It could be a potential target for anti-aging and anti-senoinflammation strategies.

\section{ACKNOWLEDGEMENTS}

This work was supported by a National Research Foundation (NRF) grant funded by the Korean government (MSIP) (Grant NO. 2018R1A2A3075425 and 2013M3A9B6076431). We thank Aging Tissue Bank (Busan, Korea) for providing aging research information.

\section{CONFLICTS OF INTEREST}

The authors have no conflicting interests.

\section{REFERENCES}

1. Chung HY, Cesari M, Anton S et al (2009) Molecular inflammation: underpinnings of aging and age-related 
diseases. Ageing Res Rev 8, 18-30

2. Chung HY, Kim DH, Lee EK et al (2018) Redefining Chronic Inflammation in Aging and Age-Related Diseases: Proposal of the Senoinflammation Concept. Aging Dis [Epub ahead of print]

3. Vasto S, Candore G, Balistreri CR et al (2007) Inflammatory networks in ageing, age-related diseases and longevity. Mech Ageing Dev 128, 83-91

4. Yu BP and Yang R (1996) Critical evaluation of the free radical theory of aging. A proposal for the oxidative stress hypothesis. Ann N Y Acad Sci 786, 1-11

5. Groslambert M and Py BF (2018) Spotlight on the NLRP3 inflammasome pathway. J Inflamm Res 11, 359-374

6. Hanouna G, Mesnard L, Vandermeersch S et al (2017) Specific calpain inhibition protects kidney against inflammaging. Sci Rep 7, 8016

7. Baker DJ, Childs BG, Durik M et al (2016) Naturally occurring p16(Ink4a)-positive cells shorten healthy lifespan. Nature 530, 184-189

8. Salminen A, Kauppinen A and Kaarniranta K (2012) Emerging role of NF-kappaB signaling in the induction of senescence-associated secretory phenotype (SASP). Cell Signal 24, 835-845

9. Young AR and Narita M (2009) SASP reflects senescence. EMBO Rep 10, 228-230

10. Coppe JP, Patil CK, Rodier F et al (2008) Senescenceassociated secretory phenotypes reveal cell-nonautonomous functions of oncogenic RAS and the p53 tumor suppressor. PLoS Biol 6, 2853-2868

11. Freund A, Orjalo AV, Desprez PY and Campisi J (2010) Inflammatory networks during cellular senescence: causes and consequences. Trends Mol Med 16, 238-246

12. Seo YH, Jung HJ, Shin HT et al (2008) Enhanced glycogenesis is involved in cellular senescence via GSK3/GS modulation. Aging Cell 7, 894-907

13. Coppe JP, Desprez PY, Krtolica A and Campisi J (2010) The senescence-associated secretory phenotype: the dark side of tumor suppression. Annu Rev Pathol 5, 99-118

14. Georgilis A, Klotz S, Hanley CJ et al (2018) PTBP1Mediated Alternative Splicing Regulates the Inflammatory Secretome and the Pro-tumorigenic Effects of Senescent Cells. Cancer Cell 34, 85-102.e109

15. Fuhrmann-Stroissnigg $\mathrm{H}$, Niedernhofer $\mathrm{L}$ ) and Robbins PD (2018) Hsp90 inhibitors as senolytic drugs to extend healthy aging. Cell Cycle 17, 1048-1055

16. Redman LM and Ravussin E (2011) Caloric restriction in humans: impact on physiological, psychological, and behavioral outcomes. Antioxid Redox Signal 14, 275-287

17. Gonzalez O, Tobia C, Ebersole J and Novak MJ (2012) Caloric restriction and chronic inflammatory diseases. Oral Dis 18, 16-31

18. Anderson RM and Weindruch R (2012) The caloric restriction paradigm: implications for healthy human aging. Am J Hum Biol 24, 101-106

19. Nadon NL, Strong R, Miller RA et al (2008) Design of aging intervention studies: the NIA interventions testing program. Age (Dordr) 30, 187-199

20. Mattson MP, Duan W, Lee J and Guo Z (2001) Suppression of brain aging and neurodegenerative disorders by dietary restriction and environmental enrichment: molecular mechanisms. Mech Ageing Dev $122,757-778$

21. Cohen DE, Supinski AM, Bonkowski MS, Donmez G and Guarente LP (2009) Neuronal SIRT1 regulates endocrine and behavioral responses to calorie restriction. Genes Dev 23, 2812-2817

22. Pahlavani MA (2000) Caloric restriction and immunosenescence: a current perspective. Front Biosci 5, D580-587

23. Kurki E, Shi J, Martonen E, Finckenberg $P$ and Mervaala $E$ (2012) Distinct effects of calorie restriction on adipose tissue cytokine and angiogenesis profiles in obese and lean mice. Nutr Metab (Lond) 9,64

24. Lijnen HR, Van Hul M and Hemmeryckx B (2012) Caloric restriction improves coagulation and inflammation profile in obese mice. Thromb Res 129, 74-79

25. Choi WH, Um MY, Ahn J, Jung CH and Ha TY (2014) Cooked rice inhibits hepatic fat accumulation by regulating lipid metabolism-related gene expression in mice fed a high-fat diet. J Med Food 17, 36-42

26. Jung KJ, Lee EK, Kim JY et al (2009) Effect of short term calorie restriction on pro-inflammatory NF-kB and AP-1 in aged rat kidney. Inflamm Res 58, 143-150

27. Sadagurski M, Landeryou T, Cady G, Bartke A, Bernal-Mizrachi E and Miller RA (2015) Transient early food restriction leads to hypothalamic changes in the long-lived crowded litter female mice. Physiol Rep 3, e12379

28. Tsutsumi A, Motoshima H, Kondo T et al (2011) Caloric restriction decreases ER stress in liver and adipose tissue in ob/ob mice. Biochem Biophys Res Commun 404, 339-344

29. Escriva F, Gavete ML, Fermin Y et al (2007) Effect of age and moderate food restriction on insulin sensitivity in Wistar rats: role of adiposity. J Endocrinol 194, 131-141

30. Zheng Y, Zhang W, Pendleton E et al (2009) Improved insulin sensitivity by calorie restriction is associated with reduction of ERK and p70S6K activities in the liver of obese Zucker rats. J Endocrinol 203, 337-347

31. Park MH, Park JY, Lee HJ et al (2013) Potent anti-diabetic effects of MHY908, a newly synthesized PPAR alpha/gamma dual agonist in $\mathrm{db} / \mathrm{db}$ mice. PLoS One 8, e78815

32. Youm YH, Nguyen KY, Grant RW et al (2015) The ketone metabolite beta-hydroxybutyrate blocks NLRP3 inflammasome-mediated inflammatory disease. Nat Med 21, 263-269

33. Fann DY, Santro T, Manzanero S et al (2014) Intermittent fasting attenuates inflammasome activity in ischemic stroke. Exp Neurol 257, 114-119

34. Traba J, Kwarteng-Siaw M, Okoli TC et al (2015) Fasting and refeeding differentially regulate NLRP3 inflammasome activation in human subjects. J Clin Invest 125 , 4592-4600

35. Chung KW, Kim DH, Park MH et al (2013) Recent advances in calorie restriction research on aging. Exp Gerontol 48, 1049-1053

36. Kim HJ, Jung KJ, Yu BP, Cho CG, Choi JS and Chung HY (2002) Modulation of redox-sensitive transcription factors by calorie restriction during aging. Mech Ageing Dev 123, 


\section{9-1595}

37. Sung B, Park S, Yu BP and Chung HY (2004) Modulation of PPAR in aging, inflammation, and calorie restriction. J Gerontol A Biol Sci Med Sci 59, 997-1006

38. Delerive P, Gervois P, Fruchart JC and Staels B (2000) Induction of IkappaBalpha expression as a mechanism contributing to the anti-inflammatory activities of peroxisome proliferator-activated receptor-alpha activators. J Biol Chem 275, 36703-36707

39. Chung SW, Kang BY, Kim SH et al (2000) Oxidized low density lipoprotein inhibits interleukin-12 production in lipopolysaccharide-activated mouse macrophages via direct interactions between peroxisome proliferator-activated receptor-gamma and nuclear factor-kappa B. J Biol Chem $275,32681-32687$

40. Chung JH, Seo AY, Chung SW et al (2008) Molecular mechanism of PPAR in the regulation of age-related inflammation. Ageing Res Rev 7, 126-136

41. Satoh A, Brace CS, Rensing N et al (2013) Sirt1 extends life span and delays aging in mice through the regulation of Nk2 homeobox 1 in the DMH and LH. Cell Metab 18, 416-430

42. Nisoli E, Tonello C, Cardile A et al (2005) Calorie restriction promotes mitochondrial biogenesis by inducing the expression of eNOS. Science 310, 314-317

43. Frescas D, Valenti L and Accili D (2005) Nuclear trapping of the forkhead transcription factor FoxO1 via Sirtdependent deacetylation promotes expression of glucogenetic genes. J Biol Chem 280, 20589-20595

44. Hong SE, Heo HS, Kim DH et al (2010) Revealing system-level correlations between aging and calorie restriction using a mouse transcriptome. Age (Dordr) 32, 15-30

45. Park D, Lee EK, Jang EJ et al (2013) Identification of the dichotomous role of age-related LCK in calorie restriction revealed by integrative analysis of cDNA microarray and interactome. Age (Dordr) 35, 1045-1060

46. Park D, Kim BC, Kim CH et al (2016) RNA-Seq analysis reveals new evidence for inflammation-related changes in aged kidney. Oncotarget 7, 30037-30048

47. He S and Sharpless NE (2017) Senescence in Health and Disease. Cell 169, 1000-1011

48. Kim CH, Lee EK, Choi YJ et al (2016) Short-term calorie restriction ameliorates genomewide, age-related alterations in DNA methylation. Aging Cell 15, 1074-1081

49. Park MH, Kim DH, Lee EK et al (2014) Age-related inflammation and insulin resistance: a review of their intricate interdependency. Arch Pharm Res 37, 1507-1514

50. Kim DH, Kim JY, Yu BP and Chung HY (2008) The activation of NF-kappaB through Akt-induced FOXO1 phosphorylation during aging and its modulation by calorie restriction. Biogerontology 9, 33-47 